\title{
Using subscales when scoring the Cushing's quality of life questionnaire
}

\author{
Jitske Tiemensma, Sarah Depaoli and John M Felt \\ Psychological Sciences, University of California, Merced, 5200 North Lake Road, Merced, California 95343, USA
}

\author{
Correspondence \\ should be addressed \\ to J Tiemensma \\ Email \\ jtiemensma@ucmerced.edu
}

\begin{abstract}
Context: Patients in long-term remission of Cushing's syndrome (CS) commonly report impaired quality of life (QoL).

The CushingQoL questionnaire is a disease-specific QoL questionnaire for patients diagnosed with CS. The developers of the CushingQoL recommend using a global (total) score to assess QoL. However, the global score does not capture all aspects of QoL as outlined by the World Health Organization (WHO).

Objective: The aim of the study was to compare the performance of different scoring options to determine the optimal method for the CushingQoL.

Design and patients: Patients in remission from CS $(n=341)$ were recruited from the Cushing's Syndrome Research Foundation's email listserv and Facebook page, and asked to complete the CushingQoL and a short demographics survey. Results: Using an exploratory analysis, adequate model fit was obtained for the global score, as well as a 2-subscale (psychosocial issues and physical problems) scoring solution. Confirmatory methods were performed to identify the optimal scoring solution. Both the global score and the 2-subscale scoring solution showed adequate model fit. However, a $\chi^{2}$ difference test indicated that the 2 -subscale scoring solution was a significantly better fit than the global score $(P<0.05)$. Conclusion: If doctors or researchers would like to tease apart physical and psychosocial issues, the 2-subscale scoring solution would be recommended, since this solution showed to be optimal in scoring the CushingQoL. Regardless of the scoring solution used, the CushingQoL has proven to be a valuable resource for assessing health-related QoL in patients with CS.
\end{abstract}

\section{Introduction}

Cushing's syndrome (CS), also known as hypercortisolism, is a rare disorder characterized by chronic overexposure to cortisol (1). CS can be caused by prolonged, high-dose glucocorticoid steroid use, adrenocorticotropic hormone (ACTH) releasing pituitary adenomas, ectopic tumors, or adrenal tumors $(1,2)$. CS is associated with a number of physical and psychological symptoms. Physical symptoms include weight gain, rounded face, violaceous striae, gonadal dysfunction, weak muscles, and easy bruising (3). Some of the psychological symptoms include depression, irritability, anxiety, and impaired quality of life (QoL) $(4,5)$. Treatment for CS depends on the cause of the hypercortisolism and typically involves cortisol-inhibiting
(C) 2016 European Society of Endocrinology Printed in Great Britain drugs, transsphenoidal surgery, adrenalectomy, radiation, or gradual reduction in the dosage of glucocorticoid steroids $(1,6,7)$. Surgery is the first-line treatment of all forms of CS, with an initial remission rate of $60-80 \%$ after transsphenoidal surgery. Adrenalectomy is the method of choice for adrenal causes of CS, as well as for ACTHdependent Cushing's disease (CD) when transsphenoidal surgery and medical treatment are unsuccessful (3). If transsphenoidal surgery for ACTH-dependent CD leaves a residual mass, radiotherapy or medical therapy might be the treatment of choice. Medical therapy is also an option when awaiting surgery or if surgery is contraindicated for all causes of CS (3). Upon remission of CS, signs and

Published by Bioscientifica Ltd 
symptoms regress substantially, although QoL typically does not return to premorbid levels $(8,9)$. Because impaired QoL is a persistent complaint from patients in remission from CS, it is important to accurately assess which aspects of QoL are affected in order to develop more effective patient interventions.

The CushingQoL questionnaire (10) is the most frequently used disease-specific QoL questionnaire for patients diagnosed with CS. Webb et al. (10) developed the CushingQoL using standardized interviews with patients and endocrinologists. The authors found the CushingQoL to be unidimensional (i.e. consisting of one global score). Although the developers mentioned subscales assessing different dimensions of QoL in the CushingQoL, Webb et al. (10) recommend the global score when assessing QoL and changes in QoL over time $(11,12,13,14)$. However, the World Health Organization (WHO) recommends that QoL be treated as a multidimensional construct containing aspects of QoL referring to physical, psychological, and social issues (15). A disadvantage of using the global score of the CushingQoL is that extracting information reflecting different dimensions of QoL becomes impossible, since all constructs are combined in the global score. While the content of the CushingQoL reflects the recommendations of the WHO, using the global score may hide impairments in QoL reflecting a specific dimension of QoL. For example, Felt et al. (16) found that using the total score of the acromegaly QoL questionnaire (a disease-specific QoL questionnaire for patients with acromegaly) could yield different conclusions about QoL than when using the subscale scores.

The current literature does not include information about which items belong to each of the subscales and subsequently, studies have only examined the psychometric properties of the global score of the CushingQoL $(10,17,18,19,20)$. While the global score has demonstrated desirable psychometric properties $(17,18,19,20)$, there have been no studies comparing alternative scoring options (i.e. different subscales) of the CushingQoL. Therefore, the aims of the present study are to i) determine if there are viable subscales in the CushingQoL, ii) if so, determine which questions belong to each of the subscales of the CushingQoL, and iii) compare the performance of the different scoring options to determine the optimal scoring solution for the CushingQoL. Given the WHO's recommendations, a 3-subscale scoring solution reflecting physical, psychological, and social aspects of QoL was hypothesized to be the best scoring solution for the CushingQoL.

\section{Subjects and methods}

\section{Participants}

Patients were invited to participate in the current study through the Cushing's Support and Research Foundation's (CSRF) listserv and Facebook page, where a message was distributed containing a web link to the study. Patients were eligible for participation if they were over 18 years of age and in remission from CS/CD. Patients were asked to complete the CushingQoL (English version) and a demographics survey. A total of 341 patients participated ( $n=25$ males, $n=305$ females, and $n=11$ unknown). The long-term treatment outcomes for these patients are described in Table 1. The protocol was approved by the University of California, Merced Institutional Review Board, and all patients provided digital informed consent before filling out the survey.

\section{Protocol}

Patients received a digital consent form first, which described the nature of the study. Once they read the

Table 1 Clinical characteristics. Data are presented as mean (s.D.) or $n(\%)$.

\begin{tabular}{lc}
\hline Clinical characteristics & Values \\
\cline { 1 - 2 } Patients with CD/CS (total $n)$ & 341 \\
Gender $(n)$ & \\
Male & 25 \\
Female & 305 \\
Unknown & 11 \\
Age (years) & $48.41(13.1)$ \\
Educational level & $12(4 \%)$ \\
No degree & $49(14 \%)$ \\
High school/GED: & $68(20 \%)$ \\
Associate's degree & $108(32 \%)$ \\
College degree & $15(4 \%)$ \\
Professional degree & $80(24 \%)$ \\
Graduate degree & $16(5 \%)$ \\
Unknown & \\
Diagnosis & $238(70 \%)$ \\
Cushing's disease & $94(28 \%)$ \\
Cushing's syndrome & $4(1 \%)$ \\
Medication induced Cushing's & $5(1 \%)$ \\
Unknown & $233(68 \%)$ \\
Transsphenoidal surgery & \\
Adrenal surgery & $66(19 \%)$ \\
Unilateral & $59(17 \%)$ \\
Bilateral & $23(7 \%)$ \\
Postoperative radiotherapy & $60(18 \%)$ \\
Recurrence & $6.88(7.4)$ \\
Duration of remission (years) & $131(38 \%)$ \\
Duration of follow-up (years) & $146(43 \%)$ \\
Hypopituitarism & $6.18 \%)$ \\
Hydrocortisone substitution & \\
Fludrocortisone & \\
\hline
\end{tabular}


form and agreed to participate, they clicked to a subsequent webpage. The second page simply asked them if they were 18 years or older. If a patient indicated they met this criterion, they then clicked to the next page, where they were asked about remission status. If the patient was in remission, then they were taken to the next webpage that included a set of instructions for how to fill out the CushingQoL questionnaire. If they understood the instructions, they could click to the next page, which included the 12 CushingQoL items as displayed in the original paper-and-pencil version published by Webb et al. (10). The survey was typed in larger font as to mimic the paper-and-pencil version, and all of the items were kept on the same page (as opposed to presenting one item at a time) to exactly mimic the paper-and-pencil version. Upon completion of the survey, patients clicked to the next page, where they received a demographics survey to complete. Given this process, the online survey would be considered as a 'minor modification' of the paper-and-pencil version (21).

\section{CushingQoL questionnaire}

The CushingQoL is a disease-specific QoL questionnaire designed to assess QoL in patients with CS (10). The questionnaire consists of 12 items measured on a fivepoint Likert-type scale assessing how often ('always' to 'never') or how much ('very much' to 'not at all') each item has been related to the patient's CS in the previous 4 weeks. The CushingQoL consists of questions reflecting dimensions of QoL related to physical aspects (e.g. 'I bruise easily'), psychological aspects (e.g. 'I am more irritable, I have sudden mood swings and angry outbursts'), and social aspects (e.g. 'I have had to give up my social or leisure activities due to my illness'). The recommended total score ranges from 0 to 100 , with a lower score indicating a greater impact on health-related QoL. The following formula can be used to calculate the total score:

$\frac{(X)-L}{H-L} \times 100$

where $X$ is the total score of the subscale of interest, $L$ is the lowest possible score of the subscale, and $H$ is the highest possible score for the subscale. This equation transforms scores to a 0 -to-100 scale, with 100 indicating the best possible QoL.

\section{Demographic survey}

We asked patients to complete 20 questions in the demographics portion of the survey: gender, age, highest level of education, race/ethnicity, marital status, height, weight, hours of sleep in a typical night, pituitary tumor (yes/no), exact diagnosis, year of diagnosis, treatment, year of treatment, recurrence (yes/no), incidence of hypopituitarism (yes/no), hydrocortisone usage (yes/no), fludrocortisone usage (yes/no), list of prescribed medications, remission (yes/no), and duration of remission. For every question, patients had the option to select 'I don't know'. The questions about race/ethnicity, marital status, height, weight, and sleep duration were not used in the present study.

\section{Statistical analysis}

Data were randomly split into two separate files in order to fully examine the possible scoring options for the CushingQoL questionnaire. The first data file $(n=120)$ was used to explore the possibility of multiple subscales existing in the CushingQoL. Specifically, an exploratory factor analysis (EFA) was used to assess possible subscales. An EFA is a statistical analysis used to determine scale dimensionality when there is little to no knowledge known about the dimensions of a measure (22). Regarding the sample size selected for this exploratory model group, it has been shown that having at least ten patients per number of items on the survey is sufficient to obtain adequate exploratory model results (22). Therefore, having 120 patients in this sample was deemed adequate. Having a larger sample size when estimating a confirmatory model will ultimately improve model estimates and minimize bias in the final model results. Therefore, the larger remaining portion of the data file $(n=221)$ was used to conduct a novel confirmatory model to assess how well the possible scoring options from the EFA fit the patterns in the data. It was important to incorporate the largest possible number of patients into the confirmatory model in order to ensure accurate model results. This confirmatory assessment was implemented through a confirmatory factor analysis (CFA). A CFA is the next logical step from an EFA when identifying possible subscales and examining different scoring options. CFAs can test hypotheses about generalizability of scale dimensions as well as compare alternative scoring options (23). When items show similarities in content, it is natural to want to group them together into different sub-groups of items. CFA is a statistical method that can be used to group items together and determine which items belong to each subscale. All analyses were conducted using Mplus version 7.3 (24). The amount of missing data was decidedly low (2\%), and the default settings in Mplus for missing data 
imputation and model identification were used (24). In order to examine the degree to which the different scoring solutions reflect the data, model fit was assessed.

Several fit measures (i.e. indices used to assess the different scoring options) are presented in the 'Results' section, each with different interpretations. The comparative fit index and Tucker Lewis index are measures of goodness of fit, where higher values (i.e. closer to 1.0) indicate better overall model fit. In contrast, the root mean square error of approximation is a badness of fit measure, and it is interpreted such that larger values indicate worse fit. Further, the Akaike information criterion, Bayesian information criterion (BIC), and sample size-adjusted BIC were used to directly compare models. In the case of each of these indices, the model associated with the lower value is deemed the statistically optimal model, although no significance test is conducted with these numbers. Finally, a $\chi^{2}$ difference test was used to directly compare different scoring solutions through a significance test. The scoring solutions revealed in the EFA were compared in the CFA by calculating a difference score in $\chi^{2}$ values. A significant $\chi^{2}$ difference value $(P<0.05)$ indicates that the model with the lower $\chi^{2}$ value fit the data significantly better than the competing model(s). Subscale correlations were calculated, and no collinearity issues were present in the data.

Potential differences in CushingQoL scores due to different causes of CS or other clinical characteristics were explored using the Mann-Whitney $U$ test. The relation between CushingQoL scores and duration of remission and duration of follow-up was explored using Spearman's correlation coefficient.

\section{Results}

\section{Patient characteristics}

Clinical characteristics of patients are detailed in Table 1. Two-hundred and thirty-eight patients (70\%) indicated to be diagnosed with CD, 94 (28\%) with CS, 4 (1\%) with medication-induced CS, and five patients were not sure what their exact diagnosis was. Out of all patients, 233 (68\%) underwent transsphenoidal surgery, and only 23 patients $(7 \%)$ received postoperative radiotherapy. One hundred and twenty-five patients underwent adrenalectomy (66 (19\%) unilateral and 59 (17\%) bilateral). All patients reported to be in remission (mean duration $6.9 \pm 7$ years) and the mean duration of follow-up after their last treatment was $8.6 \pm 8$ years. In addition, 131 patients (38\%) reported some form of hypopituitarism. A total of
146 patients (43\%) reported being on hydrocortisone substitution therapy and an additional 61 patients (18\%) reported using fludrocortisone.

\section{Exploratory factor analysis}

An EFA was conducted on the 12 QoL items with 120 patients; with a ratio of 10:1 patients/item, this sample size was sufficient for conducting such an analysis (25). Three exploratory models were estimated on the data, specifying a 1- (i.e. global score), 2-, or 3-subscale model. Results for the 1- and 2-subscale models are presented in Tables 2 and 3. Unstandardized subscale loadings and standard errors are presented for each item. These loadings can be interpreted such that relatively higher numbers represent a stronger relationship between the item and the particular subscale. Results are not presented for the 3-subscale model because one of the subscales was not statistically viable, with only a single item loading onto that subscale. Therefore, only the global score and 2-subscale scoring solution were deemed viable to investigate further.

Model fit was assessed to examine the degree to which particular scoring methods of the CushingQoL (i.e. global score and 2-subscale scoring solution) reflect response patterns exhibited in the data. Several fit measures (i.e. indices used to assess the different scoring options) are presented in Table 3, each with different interpretations described above.

Based on all of these statistical measures, the 2-subscale scoring solution was the best model. Specifically, bold

Table 2 Exploratory factor analysis (EFA) coefficients for single global score and 2-subscale models.

\begin{tabular}{|c|c|c|c|}
\hline \multirow[b]{3}{*}{ Items } & \multicolumn{3}{|c|}{ EFA coefficients } \\
\hline & \multirow[b]{2}{*}{$\begin{array}{l}\text { Single global } \\
\text { score }\end{array}$} & \multicolumn{2}{|c|}{ 2-Subscale solution } \\
\hline & & $\begin{array}{l}\text { Subscale 1: } \\
\text { psychosocial } \\
\text { issues }\end{array}$ & $\begin{array}{c}\text { Subscale 2: } \\
\text { physical } \\
\text { problems }\end{array}$ \\
\hline 09: Social & $0.814 *(0.036)$ & $0.872 *(0.078)$ & \\
\hline 10: Activities & $0.838 *(0.033)$ & $0.867 *(0.071)$ & \\
\hline 08: Going out & $0.843 *(0.031)$ & $0.827 *(0.079)$ & \\
\hline 12: Worry & $0.602 *(0.063)$ & $0.697 *(0.095)$ & \\
\hline 06: Confidence & $0.759 *(0.044)$ & $0.654 *(0.094)$ & \\
\hline 02: Pain & $0.670 *(0.054)$ & $0.622 *(0.094)$ & \\
\hline 11: Memory & $0.622 *(0.060)$ & $0.620 *(0.067)$ & \\
\hline 07: Worry appearance & $0.660 *(0.057)$ & $0.569 *(0.106)$ & \\
\hline 05: Mood & $0.724 *(0.048)$ & $0.549 *(0.097)$ & \\
\hline 03: Wound healing & $0.539 *(0.069)$ & & $0.863 *(0.077)$ \\
\hline 04: Bruising & $0.552 *(0.068)$ & & $0.720 *(0.138)$ \\
\hline 01: Sleep & $0.338 *(0.084)$ & & $0.325 *(0.119)$ \\
\hline
\end{tabular}

*Indicates significant factor loading at $P<0.05$. 
Table 3 Model fit measures for single total score and 2 -subscale solution models. Bold values indicate the betterfitting model comparing the total score model to the 2-subscale model.

\begin{tabular}{lccc}
$\begin{array}{lcc}\text { Model fit } \\
\text { measures }\end{array}$ & & Single total score & 2-Subscale solution \\
\cline { 1 - 1 } CFI & 0.843 & 0.911 \\
TLI & 0.808 & 0.863 \\
RMSEA & $0.134^{\mathrm{a}}$ & $\mathbf{0 . 1 1 3 ^ { \mathrm { b } }}$ \\
AIC & 4041.357 & $\mathbf{4 0 0 1 . 6 2 5}$ \\
BIC & 4141.707 & & $\mathbf{4 1 3 2 . 6 3 7}$ \\
Adj-BIC & 4027.892 & 3984.045
\end{tabular}

$\mathrm{CFI}$, comparative fit index, larger values indicate a better-fitting model; TLI, Tucker Lewis fit index, larger values indicate a better-fitting model; RMSEA, root mean square error of approximation, small values indicate a betterfitting model; AIC, Akaike information criterion, relatively smaller values indicate the optimal model; BIC, Bayesian information criterion, relatively smaller values indicate the optimal model; adj-BIC, sample size adjusted BIC ${ }^{\mathrm{a}} 95 \% \mathrm{CI}$ for RMSEA: 0.112, 0.157 .

b $95 \%$ Cl for RMSEA: $0.087,0.140$

model fit values in the table indicate that every index selected the 2-subscale scoring solution as being optimal compared to the global score. Although the model fit and comparison measures yielded consensus regarding the optimal scoring solution, substantive considerations were also made when evaluating the 2-subscale scoring solution. The items comprising each subscale are presented in Table 2 and appear to form substantively viable subscales related to psychosocial issues (subscale 1) and physical problems (subscale 2). The psychosocial issues subscale consists of items such as 'I have had to give up my social or leisure activities due to my illness' and 'My illness affects my everyday activities such as working or studying'. Cronbach's $\alpha$ for this subscale was high $(\alpha=0.912)$. The physical problems subscale consists of items such as 'My wounds take a long time to heal' and 'I bruise easily'. Cronbach's $\alpha$ for this subscale was also good, especially given the lower number of items in this subscale $(\alpha=0.718)$.

The next set of results presents novel confirmatory methods that were used to examine whether the 2-subscale scoring solution uncovered in the EFA can be used as a viable scoring option for assessing QoL in Cushing's patients.

\section{Confirmatory analysis}

Using confirmatory methods, the global score and the 2-subscale version identified in the EFA (Tables 2 and 3) were examined. A CFA was conducted on the 12 QoL items with 221 patients; this sample size far exceeds other confirmatory work done on this scale (20). CFA results for the global scoring solution and the 2-subscale scoring solution are presented in Tables 4 and 5. Standardized loadings $(\beta)$ are presented for each item, along with associated standard errors. These loadings represent the relative strength of the relationship between each item and the subscale. Using the same model fit measures as presented in the 'Exploratory factor analysis' section, the 2 -subscale scoring option was statistically superior compared to the total score option. Table 6 lists the items that comprise the two subscales.

In order to directly compare the two scoring options, a follow-up statistical procedure was conducted using the $\chi^{2}$ difference test. The 2-subscale model was found to be a significant improvement over the total score model $\chi_{\text {change }}^{2}(1)=63.11, \quad P<0.05$ for the CushingQoL (global model: $\chi^{2}=235.523$, degrees of freedom $=54$, $P$ value $=0.000$ and two-factor model: $\chi^{2}=172.413$, degrees of freedom $=53, P$ value $=0.000$ ); a significant $\chi^{2}$ test (i.e. $\left.P<0.05\right)$ indicates a poorly fitting model (26).

\section{CushingQoL scores}

The mean scores on the subscales as well as the global CushingQoL score is depicted in Table 7. There were no significant differences on the CushingQoL scores between patients with different diagnoses. In addition, patients with some form of hypopituitarism scored significantly lower on psychosocial issues than patients without hypopituitarism (mean score $39.7 \pm 23$ vs $48.8 \pm 23$, respectively, $U=14280, \quad P=0.001)$. Patients with

Table 4 Confirmatory factor analysis (CFA) standardized coefficients $(\beta)$ for the single global score and the 2-subscale solutions.

\begin{tabular}{|c|c|c|c|}
\hline \multirow[b]{3}{*}{ Items } & \multicolumn{3}{|c|}{ CFA coefficients } \\
\hline & \multirow[b]{2}{*}{$\begin{array}{l}\text { Single global } \\
\text { score }\end{array}$} & \multicolumn{2}{|c|}{ 2-Subscale solution } \\
\hline & & $\begin{array}{l}\text { Subscale 1: } \\
\text { psychosocial } \\
\text { issues }\end{array}$ & $\begin{array}{c}\text { Subscale 2: } \\
\text { physical } \\
\text { problems }\end{array}$ \\
\hline 10: Activities & $0.864 *(0.021)$ & $0.869 *(0.020)$ & \\
\hline 09: Social & $0.834 *(0.024)$ & $0.842 *(0.023)$ & \\
\hline 06: Confidence & $0.776 *(0.030)$ & $0.774 *(0.031)$ & \\
\hline 08: Going out & $0.767 *(0.031)$ & $0.772 *(0.030)$ & \\
\hline 07: Worry appearance & $0.749 *(0.033)$ & $0.750 *(0.033)$ & \\
\hline 02: Pain & $0.717 *(0.036)$ & $0.716 *(0.036)$ & \\
\hline 11: Memory & $0.659 *(0.041)$ & $0.654 *(0.041)$ & \\
\hline 12: Worry & $0.649 *(0.042)$ & $0.651 *(0.041)$ & \\
\hline 05: Mood & $0.576 *(0.048)$ & $0.568 *(0.049)$ & \\
\hline 03: Wound healing & $0.633 *(0.043)$ & & $0.883 *(0.036)$ \\
\hline 04: Bruising & $0.474 *(0.055)$ & & $0.701 *(0.043)$ \\
\hline 01: Sleep & $0.489 *(0.054)$ & & $0.510 *(0.059)$ \\
\hline
\end{tabular}

*Indicates significant factor loading at $P<0.05$ 
Table 5 Model fit measures for single total score and 2-subscale solutions. Bold values indicate the better-fitting model comparing the total score model to the 2-subscale model.

\begin{tabular}{|c|c|c|}
\hline Model fit measures & Single total score & 2-Subscale solution \\
\hline CFI & 0.875 & 0.917 \\
\hline TLI & 0.847 & 0.897 \\
\hline RMSEA & $0.123^{a}$ & $0.101^{b}$ \\
\hline AIC & 7359.007 & 7297.897 \\
\hline $\mathrm{BIC}$ & 7481.341 & 7423.629 \\
\hline Adj-BIC & 7367.255 & 7306.374 \\
\hline
\end{tabular}

$\mathrm{CFI}$, comparative fit index, larger values indicate a better-fitting model; TLI, Tucker Lewis fit index, larger values indicate a better-fitting model; RMSEA, root mean square error of approximation, small values indicate a betterfitting model; AIC, Akaike information criterion, relatively smaller values indicate the optimal model; BIC, Bayesian information criterion, relatively smaller values indicate the optimal model; adj-BIC, sample-size-adjusted BIC a $95 \%$ CI for RMSEA: 0.107, 0.140.

${ }^{b} 95 \%$ Cl for RMSEA: $0.084,0.118$.

hypopituitarism also scored significantly lower on the physical problems subscale compared with patients without hypopituitarism (mean score $44.2 \pm 25$ vs $56.1 \pm 22$, respectively, $U=14$ 757.5, $P<0.001$ ). Lastly, patients with hypopituitarism scored significantly lower on the global CushingQoL score than patients without hypopituitarism (mean score $40.9 \pm 22$ vs $50.7 \pm 21$, respectively, $U=14621$, $P<0.001)$. A pattern similar to patients with vs without hypopituitarism emerged when exploring possible differences in CushingQoL scores in patients with and without hydrocortisone usage. Patients with hydrocortisone usage scored significantly lower on psychosocial issues (mean score $35.6 \pm 22)$ than patients who did not use hydrocortisone (mean score 50.7 $\pm 22, U=18452.5, P<0.001$ ). Patients using hydrocortisone also scored lower on the physical problems subscale compared with patients who did not report using hydrocortisone (mean score $47.8 \pm 24$ vs $56.9 \pm 22$, respectively, $U=17847.5, P<0.001)$. On the global CushingQoL score, patients with hydrocortisone usage (mean score $37.3 \pm 21$ ) scored significantly lower than patients without hydrocortisone usage $(52.2 \pm 20$, $U=18$ 711, $P<0.001$ ).

Lastly, the relationship between CushingQoL scores and duration of remission and follow-up was explored. There was a positive association between duration of remission and the psychosocial issues subscale $(r=0.193$, $P=0.001)$ and the global CushingQoL score $(r=0.144$, $P=0.012$ ), indicating that a longer duration of remission is related to a better score on the psychosocial issues subscale and global CushingQoL score. Furthermore, there was a positive association between duration of follow-up and psychosocial issues $(r=0.141, P=0.010)$, which means that a longer duration of follow-up was related to a better score on the psychosocial issues subscale.

\section{Discussion}

This is the first study to fully examine and report different scoring options for the CushingQoL questionnaire. Exploratory methods revealed two possible subscales of the CushingQoL: a psychosocial issues subscale and a physical problems subscale. Subsequently, the confirmatory methods indicated that these two subscales were optimal compared to the global CushingQoL score.

Using an exploratory analysis, the global CushingQoL score, a 2-subscale scoring solution and a 3-subscale scoring solution were explored. Adequate model fit was obtained for the global score, as well as the 2-subscale scoring solution. However, statistical findings indicated that the 3-subscale scoring solution was not viable. Given these findings, confirmatory methods were performed on a new sample of patients to identify the optimal scoring solution. Both the global score and the 2-subscale scoring solution showed adequate model fit. Results obtained for the global score were comparable to a previous confirmatory investigation examining the global CushingQoL score (20). However, the 2-subscale scoring solution outperformed the global CushingQoL score on all statistical indices implemented in the current investigation. Therefore, it is recommend to use the 2-subscale scoring solution when using the CushingQoL.

The 2-subscale scoring solution yielded a psychosocial issues subscale and a physical problems subscale. The items in each of these subscales are detailed in Table 6 .

Table 6 Items in each subscale of the CushingQoL.

Psychosocial issues subscale

2. I have pain that keeps me from leading a normal life

5. I am more irritable, I have sudden mood swings and angry outbursts

6. I have less self-confidence, I feel more insecure

7. I am worried about the changes in my physical appearance due to my illness

8. I feel less like going out or seeing relatives or friends

9. I had to give up my social or leisure activities due to my illness

10. My illness affects my everyday activities such as working or studying

11. It is difficult for me to remember things

12. I am worried about my health in the future

Physical problems subscale

1. I have trouble sleeping

3. My wounds take a long time to heal

4. I bruise easily 
Table 7 Example global CushingQoL and subscale scores.

\begin{tabular}{|c|c|c|c|}
\hline Participants & $\begin{array}{l}\text { Psychosocial } \\
\text { issues }\end{array}$ & $\begin{array}{l}\text { Physical } \\
\text { problems }\end{array}$ & $\begin{array}{c}\text { Global } \\
\text { CushingQoL } \\
\text { score }\end{array}$ \\
\hline Entire sample $(n=341)$ & $\begin{array}{c}43.89 \\
\text { (s.D. }=23.7)\end{array}$ & $\begin{array}{c}50.66 \\
\text { (s.D. }=24.2)\end{array}$ & $\begin{array}{c}45.54 \\
\text { (s.D. }=21.9)\end{array}$ \\
\hline Participant no. 51 & 0.00 & 25.00 & 6.25 \\
\hline Participant no. 52 & 69.44 & 33.33 & 60.42 \\
\hline Participant no. 63 & 36.11 & 33.33 & 35.42 \\
\hline Participant no. 144 & 11.11 & 50.00 & 20.83 \\
\hline Participant no. 146 & 0.00 & 50.00 & 12.50 \\
\hline Participant no. 148 & 91.67 & 58.33 & 83.33 \\
\hline Participant no. 167 & 55.56 & 58.33 & 56.25 \\
\hline Participant no. 284 & 33.33 & 91.67 & 47.92 \\
\hline Participant no. 285 & 97.22 & 100.00 & 97.92 \\
\hline
\end{tabular}

Bold scores represent cases where the global CushingQoL score conflicts with the 2-subscale scoring solution.

To better interpret the scoring of the subscales, the same standardization can be used as proposed by the developers of the CushingQoL. This standardization can also be used for the global score of the CushingQoL, keeping both subscales and the global score on the same metric (10).

The WHO recommends that QoL be treated as a multidimensional construct containing aspects of QoL referring to physical, psychological, and social issues (15). Using the 2-subscale scoring solution reflects the WHO's recommendations better than the global score. The global score collapses QoL across all dimensions, which makes it impossible to disentangle physical problems and psychosocial issues. The two proposed subscales make it possible for doctors and researchers to gain more insight in both physical problems and psychosocial issues.

To illustrate the potential clinical benefit of using the 2-subscale scoring option of the CushingQoL, a sample of nine scores is presented in Table 7. As an example, patient no. 148 received a global CushingQoL score of 83.33, which represents a relatively high QoL score. However, upon examination of the subscale scores, it was revealed that patient no. 148 received a relatively high score for the psychosocial issues subscale of 91.67, but the physical problems subscale score was rather low at 58.33. While patient no. 148 exhibited a relatively high overall QoL, this global score was not able to capture the impairment this person was experiencing regarding physical problems. Taking the two subscales into account when assessing QoL is important, especially when discrepancies between the global and subscale scores are present. It should be noted that a QoL questionnaire could never substitute a complete clinical evaluation. A QoL assessment should always be used in conjunction with a clinical evaluation, not as a stand-alone tool. However, having more information about different aspects of QoL (as defined by the WHO) will help clinical practitioners in better assessing, diagnosing, and helping patients. If practitioners would like to tease apart different aspects of QoL, it is recommended to use the two subscales. However, if they want a quick overview of patients' global QoL, the global CushingQoL score could be used.

The present study has some limitations. First, because the data were collected online, we were unable to verify if all respondents were in fact CS patients. A call for participants was sent out through a patient association listserv and their Facebook page, and was also linked to the patient association website, which presumably does not attract non-Cushing's patients. Furthermore, specific screening questions were used to determine eligibility. A second limitation is the lack of complementary clinical information from medical files, to corroborate the veracity of clinical data provided by patients. A third limitation is the proper assessment of the digital version of the CushingQoL. The CushingQoL was translated from the original paper-and-pencil format to the online format implemented here, which could be thought of as a limitation or threat to validity. The translation process was conducted in accordance with the guidelines set forth in the ISPOR ePRO good research practices task force report (21). However, one limitation here was that only a single patient was conferred with regarding a cognitive interview about the survey. Coons et al. (21) recommend that five to ten patients be interviewed during this portion of the assessment. Future work should focus in more detail on the conversion of the CushingQoL to electronic formatting in order to preserve the validity and psychometric properties of the original paper-and-pencil survey developed by Webb et al. (10). Lastly, the recruitment through a patient association might have introduced a bias in respondents, since not all patients in routine medical care join a patient association.

In conclusion, the CushingQoL shows good psychometric properties. Both the global CushingQoL score and the 2-subscale scoring solution showed adequate model fit. If doctors or researchers would like to tease apart physical and psychosocial issues, the 2-subscale scoring solution would be recommended, since this solution showed to be optimal in scoring the CushingQoL. Regardless of the scoring solution used, the CushingQoL has proven to be a valuable resource for assessing healthrelated QoL in patients with CS. 


\section{Declaration of interest}

The authors declare that there is no conflict of interest that could be perceived as prejudicing the impartiality of the research reported.

Funding

This research did not receive any specific grant from any funding agency in the public, commercial or not-for-profit sector.

\section{Acknowledgements}

We would like to thank the Cushing's Support and Research Foundation (CSRF) and acknowledge Karen Campbell's support and help in collecting the data. We would also like to thank all the patients who participated in this study.

\section{References}

1 Bista B \& Beck N. Cushing syndrome. Indian Journal of Pediatrics 201481 158-164. (doi:10.1007/s12098-013-1203-8)

2 Lacroix A, Feelders RA, Stratakis CA \& Nieman LK. Cushing's syndrome. Lancet 2015386 913-927. (doi:10.1016/S0140-6736(14)61375-1)

3 Sharma ST, Nieman LK \& Feelders RA. Cushing's syndrome: epidemiology and developments in disease management. Clinical Epidemiology 20157 281-293. (doi:10.2147/CLEP.S44336)

4 Tiemensma J, Biermasz NR, Middelkoop HA, van der Mast RC, Romijn JA \& Pereira AM. Increased prevalence of psychopathology and maladaptive personality traits after long-term cure of Cushing's disease. Journal of Clinical Endocrinology and Metabolism 201095 E129-E141. (doi:10.1210/jc.2010-0512)

5 Pereira AM, Tiemensma J \& Romijn JA. Neuropsychiatric disorders in Cushing's syndrome. Neuroendocrinology 201092 (Suppl 1) 65-70. (doi:10.1159/000314317)

6 Biller BMK, Grossman AB, Stewart PM, Melmed S, Bertagna X, Bertherat J, Buchfelder M, Colao A, Hermus AR, Hofland LJ et al. Treatment of adrenocorticotropin-dependent Cushing's syndrome: a consensus statement. Journal of Clinical Endocrinology and Metabolism 200893 2454-2462. (doi:10.1210/jc.2007-2734)

7 Chow JT, Thompson GB, Grant CS, Farley DR, Richards ML \& Young WF. Bilateral laparoscopic adrenalectomy for corticotrophin-dependent Cushing's syndrome: a review of the Mayo Clinic experience. Clinical Endocrinology 200868 513-519. (doi:10.1111/j.1365-2265.2007.03082.x)

8 Ragnarsson O \& Johannsson G. Cushing's syndrome: a structured shortand long-term management plan for patients in remission. European Journal of Endocrinology 2013169 R139-R152. (doi:10.1530/EJE-13-0534)

9 Santos A, Crespo I, Aulinas A, Resmini E, Valassi E \& Webb SM. Quality of life in Cushing's syndrome. Pituitary 201518 195-200. (doi:10.1007/ s11102-015-0640-y)

10 Webb SM, Badia X, Barahona MJ, Colao A, Strasburger CJ, Tabarin A, van Aken MO, Pivonello R, Stalla G, Lamberts SWJ et al. Evaluation of health-related quality of life in patients with Cushing's syndrome with a new questionnaire. European Journal of Endocrinology 2008158 623-630. (doi:10.1530/EJE-07-0762)

11 Tiemensma J, Kaptein AA, Pereira AM, Smit JW, Romijn JA \& Biermasz NR. Negative illness perceptions are associated with impaired quality of life in patients after long-term remission of Cushing's syndrome. European Journal of Endocrinology 2011165 527-535 (doi:10.1530/EJE-11-0307)

12 Valassi E, Santos A, Yaneva M, Tóth M, Strasburger CJ, Chanson P, Wass JA, Chabre O, Pfeifer M, Feelders RA et al. The European Registry on Cushing's syndrome: 2-year experience. Baseline demographic and clinical characteristics. European Journal of Endocrinology 2011165 383-392. (doi:10.1530/EJE-11-0272)

13 Webb SM, Ware JE, Forsythe A, Yang M, Badia X, Nelson LM, Signorovitch JE, McLeod L, Maldonado M, Zgliczynski W et al. Treatment effectiveness of pasireotide on health-related quality of life in patients with Cushing's disease. European Journal of Endocrinology 2014171 89-98. (doi:10.1530/EJE-13-1013)

14 Carluccio A, Sundaram NK, Chablani S, Amrock LG, Lambert JK, Post KD \& Geer EB. Predictors of quality of life in 102 patients with treated Cushing's disease. Clinical Endocrinology 201582 404-411. (doi:10.1111/cen.12521)

15 The WHOQOL Group. The World Health Organization quality of life assessment (WHOQOL): position paper from the World Health Organization. Social Science \& Medicine 199541 1403-1409. (doi:10.1016/0277-9536(95)00112-K)

16 Felt JM, Depaoli S, Pereira AM, Biermasz NR \& Tiemensma J. Total score or subscales in scoring the acromegaly quality of life questionnaire (AcroQoL): using novel confirmatory methods to compare scoring options. European Journal of Endocrinology 2015173 37-42. (doi:10.1530/EJE-15-0228)

17 Badia X, Roset M, Valassi E, Franz H, Forsythe A \& Webb SM. Mapping CushingQOL scores to EQ-5D utility values using data from the European Registry on Cushing's syndrome (ERCUSYN). Quality of Life Research 201322 2941-2950. (doi:10.1007/s11136-013-0396-7)

18 Santos A, Resmini E, Martínez-Momblán MA, Crespo I, Valassi E, Roset M, Badia X \& Webb SM. Psychometric performance of the CushingQoL questionnaire in conditions of real clinical practice. European Journal of Endocrinology 2012167 337-342. (doi:10.1530/EJE-12-0325)

19 Roset M, Badia X, Forsythe A \& Webb SM. Mapping CushingQoL scores onto SF-6D utility values in patients with Cushing's syndrome. Patient 20136 103-111. (doi:10.1007/s40271-013-0010-7)

20 Nelson LM, Forsythe A, McLeod L, Pulgar S, Maldonado M, Coles T, Zhang Y, Webb SM \& Badia X. Psychometric evaluation of the Cushing's quality-of-life questionnaire. Patient 20136 113-124. (doi:10.1007/s40271-013-0012-5)

21 Coons SJ, Gwaltney CJ, Hays RD, Lundy JJ, Sloan JA, Revicki DA, Lenderking WR, Cella D \& Basch E. Recommendations on evidence needed to support measurement equivalence between electronic and paper-based patient-reported outcome (PRO) measures: ISPOR ePRO Good Research Practices Task Force report. Value in Health 200912 419-429. (doi:10.1111/j.1524-4733.2008.00470.x)

22 Furr R \& Bacharach V. Test dimensionality and factor analysis Psychometrics: An Introduction. 2nd edn, pp. 71-97, Thousand Oaks, CA, USA: SAGE Publications, 2013.

23 Furr R \& Bacharach V. Confirmatory factor analysis Psychometrics: An Introduction. 2nd edn, pp. 331-353, Thousand Oaks, CA, USA: SAGE Publications, 2013.

24 Muthén L \& Muthén B. Mplus User's Guide, 7th edn, 2014.

25 Nunnally JC. Psychometric Theory, 7th edn, Los Angeles, CA: Muthén \& Muthén, 1998-2014.

26 Bentler PM \& Bonett DG. Significance tests and goodness of fit in the analysis of covariance structures. Psychological Bulletin $1980 \mathbf{8 8}$ 588-606. (doi:10.1037/0033-2909.88.3.588)
Received 28 June 2015

Revised version received 25 September 2015

Accepted 2 October 2015 